\title{
Sodium Tetradecyl Sulfate
}

National Cancer Institute

\section{Source}

National Cancer Institute. Sodium Tetradecyl Sulfate. NCI Thesaurus. Code C66555.

An anionic surfactant used as a sclerosing agent. Upon injection into a varicose vein, sodium tetradecyl sulfate (STS) interacts with lipids on the vein walls thereby causing intimal inflammation, destruction of the vein, and thrombus and fibrous tissue formation.

This eventually leads to occlusion of the injected vein. 